\title{
Control of an Active Suspension System as a Benchmark for Design and Optimization of Restricted-Complexity Controllers
}

\author{
I. D. Landau ${ }^{\dagger}$, A. Karimi ${ }^{\ddagger}$, L. Mišković ${ }^{\ddagger}$ and H. Prochazka ${ }^{\dagger}$ \\ $\dagger$ Laboratoire d'Automatique de Grenoble, ENSIEG, INPG, B.P. 46, 38402 \\ Saint Martin d'Hères , France \\ † Laboratoire d'Automatique, Ecole Polytechnique Fédérale de Lausanne \\ (EPFL), CH-1015 Lausanne, Switzerland
}

\begin{abstract}
A benchmark problem for restricted complexity controller design is introduced. The objective is to design the lowest-order controller which meets the control specifications for an active suspension system. The input-output data of the plant are provided on the benchmark site and the final controllers are evaluated using the closed-loop data. Thirteen solutions proposed to solve the benchmark problem are briefly presented and classified in terms of methodology and compared with respect to their complexity and performance.
\end{abstract}

Keywords: Active suspension, benchmark, restricted-order controller

\section{Introduction}

The problem of restricted-complexity controller design is theoretically very challenging and has a great importance in practice. Many approaches for solving this problem can be found in the literature and it is not an easy task to select one for a specific application. Experiences have shown that benchmark problems are efficient for comparing different approaches allowing to assess their potential and weakness.

In this paper, the control of an active suspension system is considered as a benchmark for restricted complexity controller design. The benchmark problem contains all the steps of a control design problem from data acquisition, identification, robust controller design to controller evaluation and final tuning with experiments on the real system. The plant model is of very high-order and the control specifications are in terms of the constraints on closed-loop sensitivity functions. So, the design of a non-restricted complexity controller is a classical control problem. However, in this benchmark, the main challenge is to design the simplest controller which meets the control specifications.

The benchmark problem was launched on November 2001 together with a call for papers for a Special Issue on "Design and Optimization of Restricted Complexity Controllers" [14]. Several solutions to the benchmark problem were proposed by different 
research groups from all over the world. The participants to the benchmark presented their works in a workshop held at Grenoble on January 2003 [15]. In this paper, the benchmark problem is presented and the solutions are compared in terms of different criteria.

The paper is organized as follows: Section 2 presents the benchmark problem containing system description, identified models, control specifications and control evaluation. The participants to the benchmark are introduced briefly in Section 3. The designs are compared with respect to different criteria in Section 4. Finally, Section 5 gives some concluding remarks.

\section{Benchmark Problem}

The active suspension system is a laboratory set-up at Laboratoire d'Automatique de Grenoble (INPG/CNRS) and uses an active suspension manufactured by Hutchinson. For this benchmark the problem of attenuation of disturbances in a large frequency band according to given control specifications has been considered.

To help the participants, the organizers have provided on the web site of the Benchmark input-output data for model identification, as well as an identified discrete-time high-order model of the plant and a continuous-time model [14]. The use of these models for controller design is arbitrary, and it was clearly stated that none of them are considered to be the exact model of the plant. One can either use them or try to find another appropriate model by data acquisition on the real system. Experiments on the platform (data acquisition or controller testing) have been carried out by the staff of Laboratoire d'Automatique de Grenoble either on the basis of the protocols defined in the benchmark or using the protocols defined by the participants. One of the objectives of the benchmark was to allow participants to collect data from the system, design the controller on the basis of acquired data and test the controller in real time. The authors were advised to design and tune their controllers using the models (provided or identified) because only 4 experiments on the real system were allowed for each participant.

\subsection{System Description}

The active suspension system set-up shown in Fig. 1 is used to test in this benchmark the disturbance attenuation in a large frequency band. Fig. 2 shows the scheme of the active hydro-suspension system used to reduce the machine vibrations. The principal parts of the active suspension system are:

- an elastomere cone that encloses the main chamber filled with silicon oil (1);

- an inertia chamber enclosed with a flexible membrane (2);

- a piston (3) that is fixed on a DC motor. When the position of the piston is fixed, the suspension system is passive;

- an orifice (4) that allows oil flow between two chambers.

The principal idea of the active suspension is to change the elasticity of the system in order to absorb the vibrations generated by the machine that we want to isolate. For the 

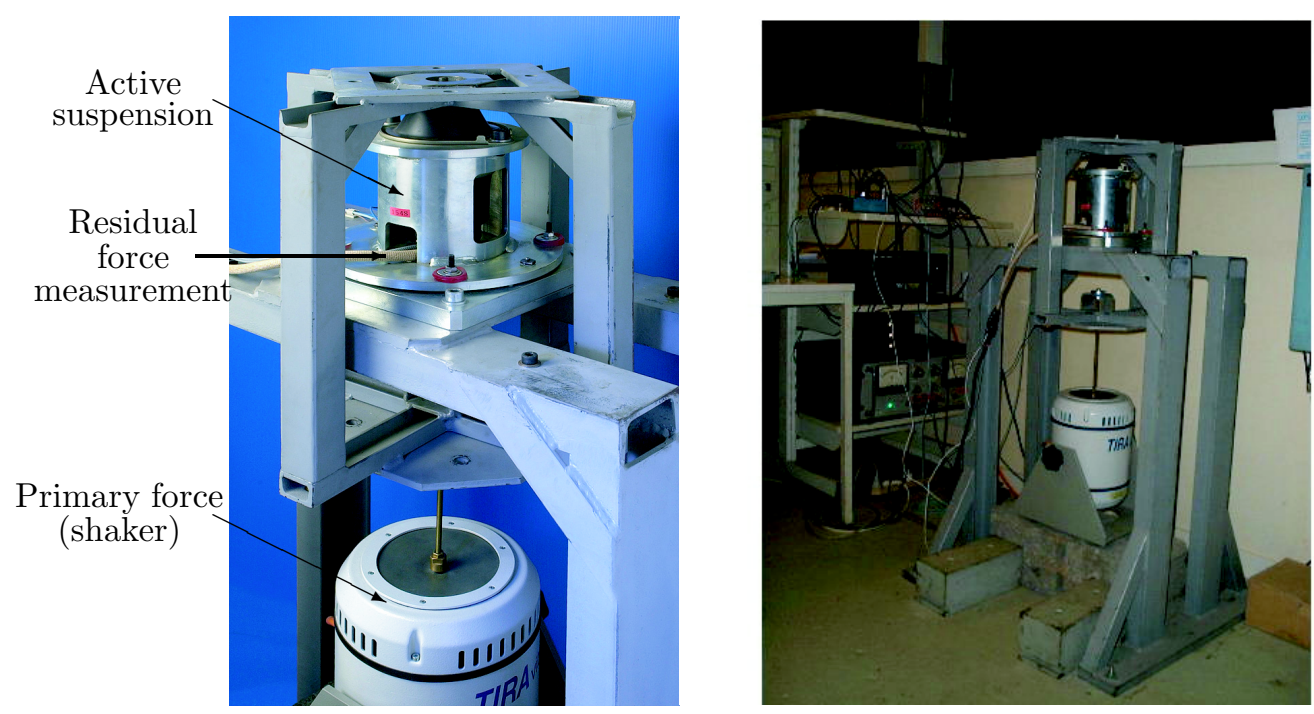

Figure 1: Photos of the active suspension system. Courtesy of Hutchinson Research Center and Laboratoire d'Automatique de Grenoble (INPG/CNRS)

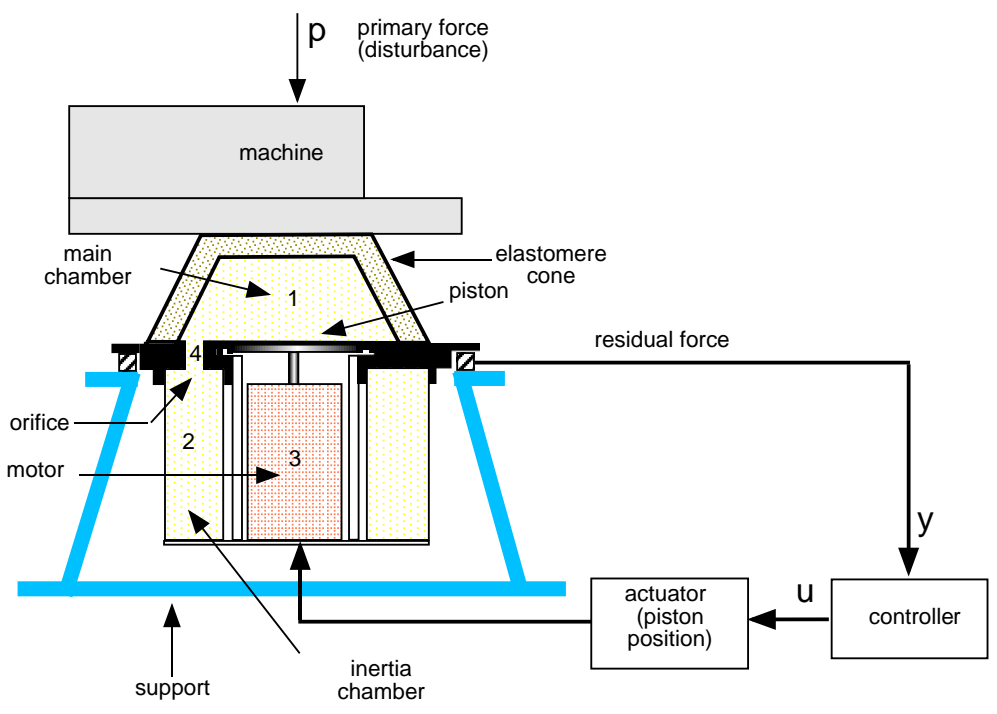

Figure 2: The schematic diagram of the active suspension system 


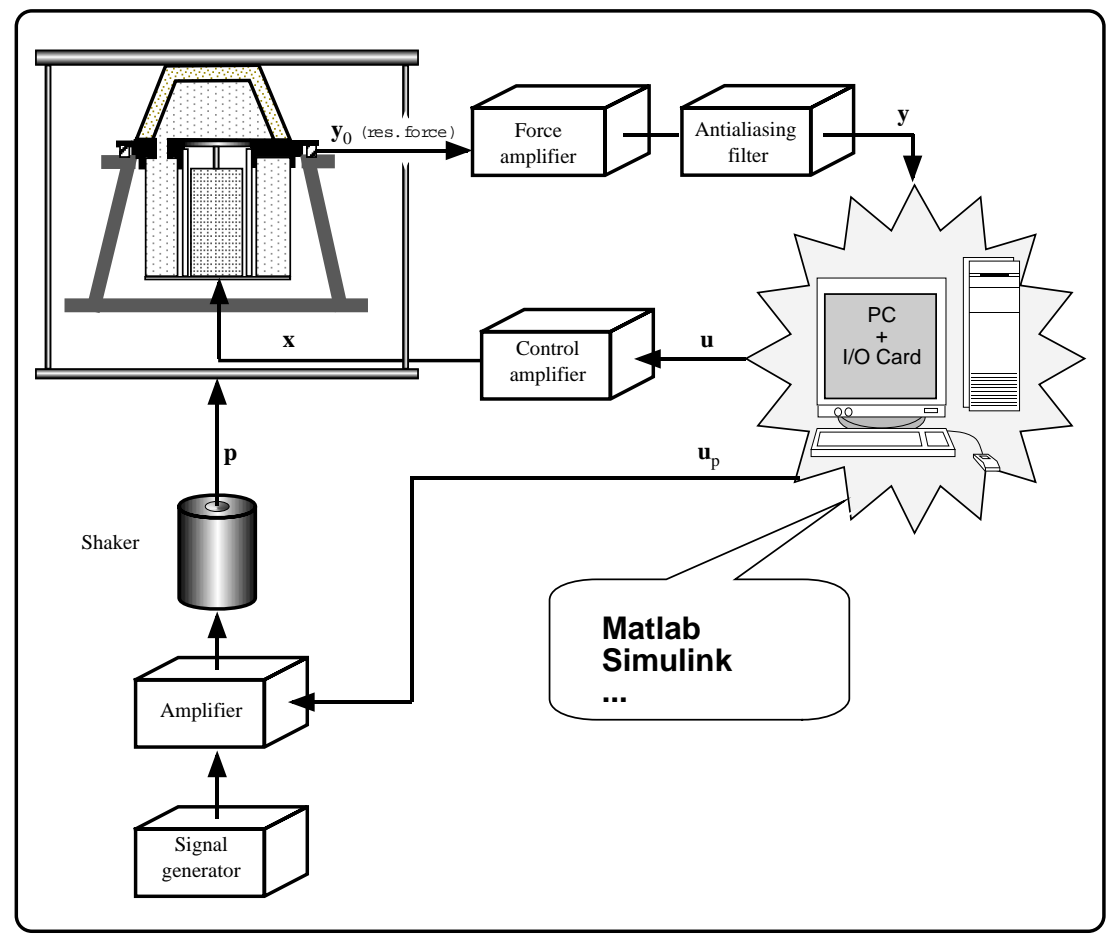

Figure 3: Block diagram of the data acquisition system

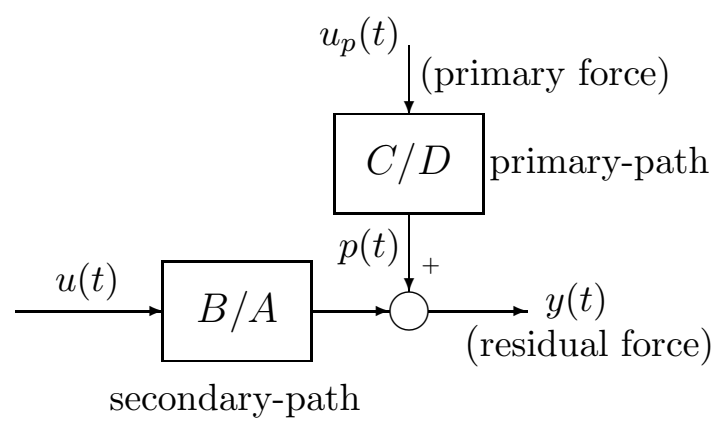

Figure 4: Block diagram of the active suspension system

experimental purposes the machine is replaced by a shaker which is driven by a computer generated control signal. The output of the system is the measured voltage corresponding to the residual force. The control input drives the position of the piston via an actuator. The system is controlled by a PC via a data acquisition system (see Fig. 3). The sampling frequency is a design parameter (upper limit: $1 \mathrm{kHz}$ ).

\subsection{Identified models}

The block diagram of the active suspension system is presented in Fig. 4. The transfer function between the excitation of the shaker and the residual force is called the primary path $(\mathrm{C} / \mathrm{D})$. The secondary path is defined as the transfer function between the control input and the residual force (B/A). Two models for the system are identified, corresponding to the primary and secondary path. Here $u_{p}(t)$ denotes the input of the primary path 


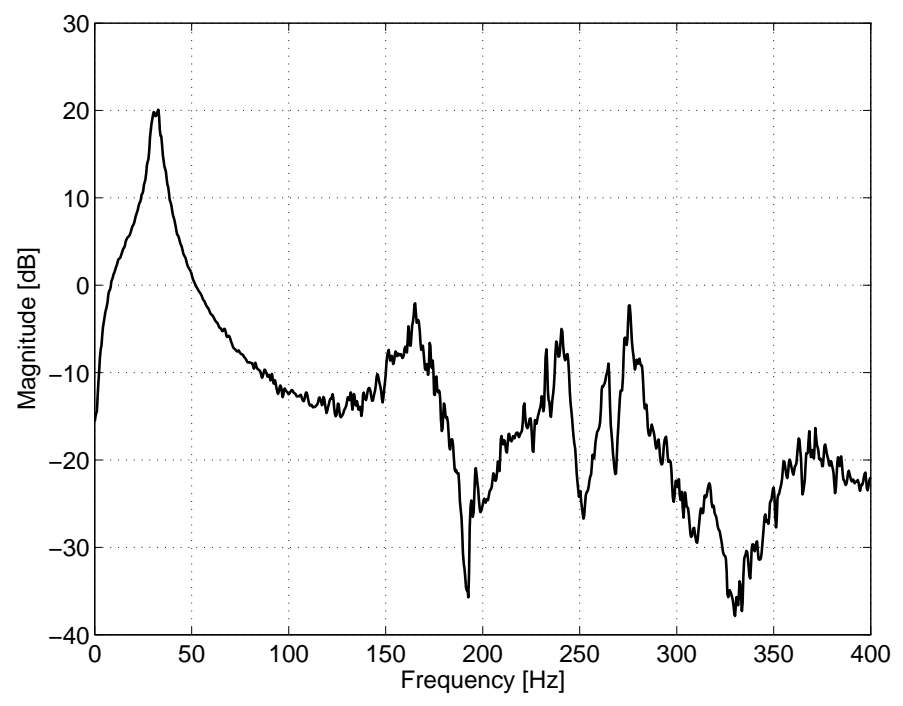

Figure 5: Frequency characteristic of the non-parametric model of the primary path

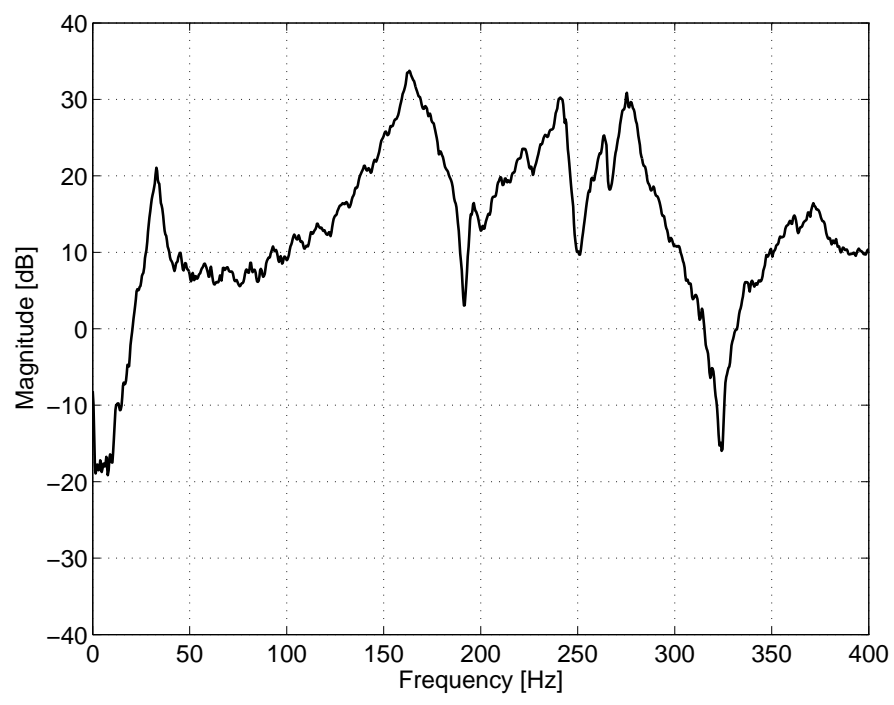

Figure 6: Frequency characteristic of the non-parametric model of the secondary path

(excitation of the shaker), $u(t)$ is the input of the secondary path (proportional to the piston position) and $y(t)$ is the system output (residual force). The sampling frequency for data acquisition is chosen to be $F_{s}=800 \mathrm{~Hz}$.

\subsubsection{Non-parametric model}

A non parametric model of the primary path can be identified by the spectral analysis method. This analysis shows that the model contains a high-resonant mode at frequency about $31,5 \mathrm{~Hz}$. The magnitude of the frequency response of the primary path is given in Fig. 5. The magnitude of the frequency response of the secondary path can also be computed using spectral analysis (see Fig. 6). 


\subsubsection{Discrete-time model}

Primary path model: To identify the primary path model $(C / D)$ the shaker is excited by a PRBS using a 10-bit shift register with a clock frequency of $F_{s} / 2$.

$$
\begin{aligned}
& C\left(z^{-1}\right)=c_{1} z^{-1}+\cdots c_{n_{C}} z^{-n_{C}} \\
& D\left(z^{-1}\right)=1+d_{1} z^{-1}+\cdots+d_{n_{D}} z^{-n_{D}}
\end{aligned}
$$

The adopted number of parameters of the polynomials $C\left(z^{-1}\right)$ and $D\left(z^{-1}\right)$ are $n_{C}=$ 8 and $n_{D}=12$, respectively.

Secondary path model: To identify the secondary path model $(B / A)$ the input $u$ is excited by the same PRBS with a clock frequency of $F_{s} / 4$.

$$
\begin{aligned}
& B\left(z^{-1}\right)=b_{1} z^{-1}+\cdots b_{n_{B}} z^{-n_{B}} \\
& A\left(z^{-1}\right)=1+a_{1} z^{-1}+\cdots+a_{n_{A}} z^{-n_{A}}
\end{aligned}
$$

The adopted number of parameters of the polynomials $A\left(z^{-1}\right)$ and $B\left(z^{-1}\right)$ are $n_{A}=$ 14 and $n_{B}=16$, respectively.

The model parameters can be downloaded from the Benchmark site [14]. Note the existence of a double differentiator in the secondary path model. These models have been provided only as a guideline and it is not claimed that they are the exact models or the best models which can be identified from data.

\subsubsection{Continuous-time model}

The continuous-time models of the plant have been obtained by conversion of discrete-time models using the zero-order hold method.

Fig. 7 shows the magnitude of the frequency response of the secondary path models obtained from spectral analysis, identified discrete-time model and continuous-time model. It can be observed that the discrete-time model and non-parametric model have very close frequency response.

\subsection{Control Specifications}

The non parametric model of the primary path obtained by the spectral analysis method shows that there are several vibration modes, whereas the first mode around $31,5 \mathrm{~Hz}$ is the most important. The second vibration mode around $160 \mathrm{~Hz}$ should also be taken into consideration when designing the controller.

Control objective: The goal is to compute a linear discrete-time controller which minimizes the residual force around the first and second vibration modes of the primary path model and to try to distribute the amplification of the disturbances over the higher frequencies. In addition, the controller gain should be equal to zero at the frequency of $F_{s} / 2$ (the term $1+z^{-1}$ should be incorporated in the controller).

More precisely, the control objective can be presented in terms of the constraints for the closed-loop sensitivity functions. The structure of the closed-loop system is given in Fig. 8, where $K=R / S$ is the controller transfer function:

$$
\begin{aligned}
R\left(z^{-1}\right) & =r_{0}+r_{1} z^{-1}+\cdots r_{n_{r}} z^{-n_{r}} \\
S\left(z^{-1}\right) & =1+s_{1} z^{-1}+\cdots+s_{n_{s}} z^{-n_{s}}
\end{aligned}
$$




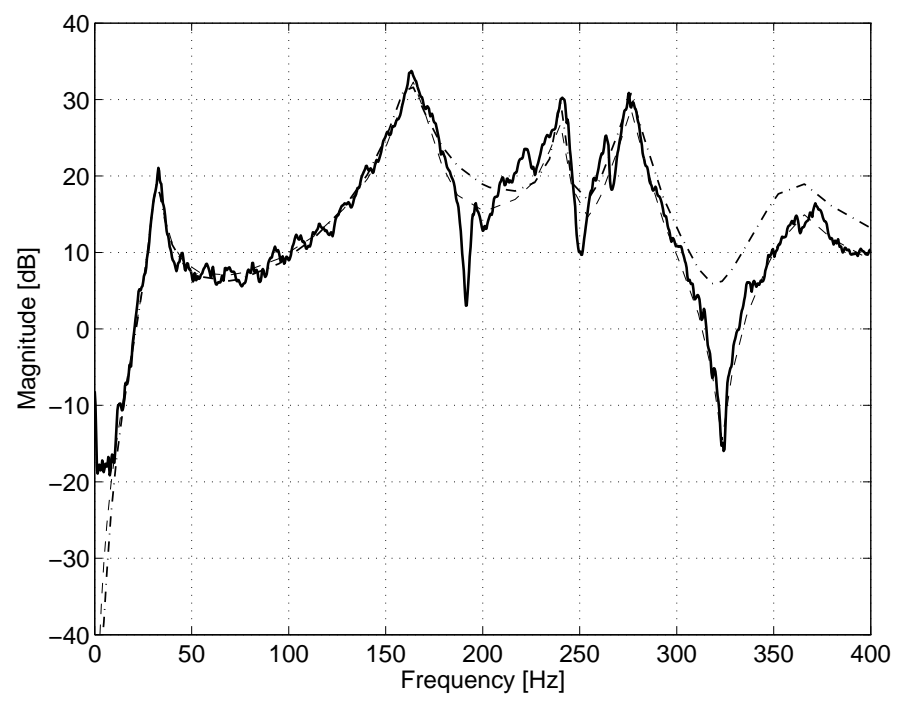

Figure 7: Frequency characteristics of the non-parametric model (solid), discrete-time model (dashed) and continuous-time model (dashed-dot)

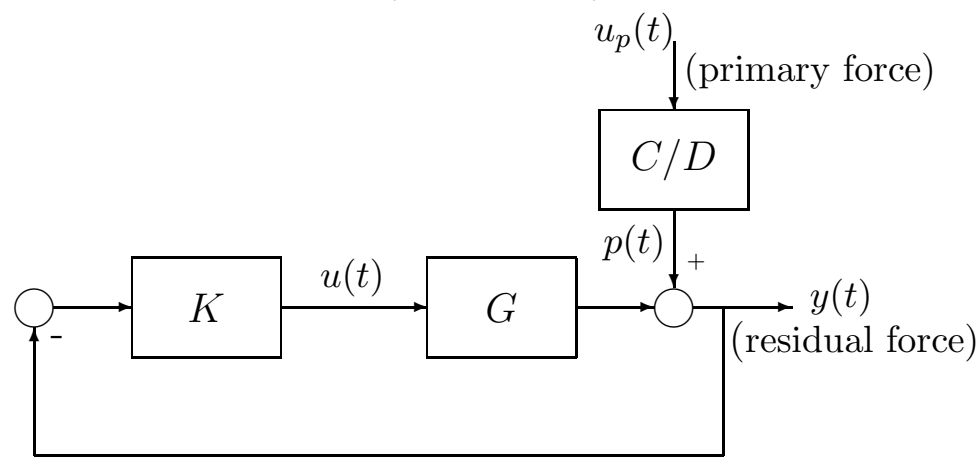

Figure 8: Block diagram of the closed-loop control system

and $G=B / A$ is the secondary path model.

Let the output and input sensitivity functions be defined as follows:

Output sensitivity function:

$$
S_{y p}\left(z^{-1}\right)=\frac{1}{1+K G}=\frac{A\left(z^{-1}\right) S\left(z^{-1}\right)}{P\left(z^{-1}\right)}
$$

\section{Input sensitivity function:}

$$
S_{u p}\left(z^{-1}\right)=-\frac{K}{1+K G}=\frac{A\left(z^{-1}\right) R\left(z^{-1}\right)}{P\left(z^{-1}\right)}
$$

where $P\left(z^{-1}\right)=A\left(z^{-1}\right) S\left(z^{-1}\right)+B\left(z^{-1}\right) R\left(z^{-1}\right)$ is the closed-loop characteristic polynomial. The constraints on the output and input sensitivity functions are shown in Fig. 9 and Fig. 10, respectively. 


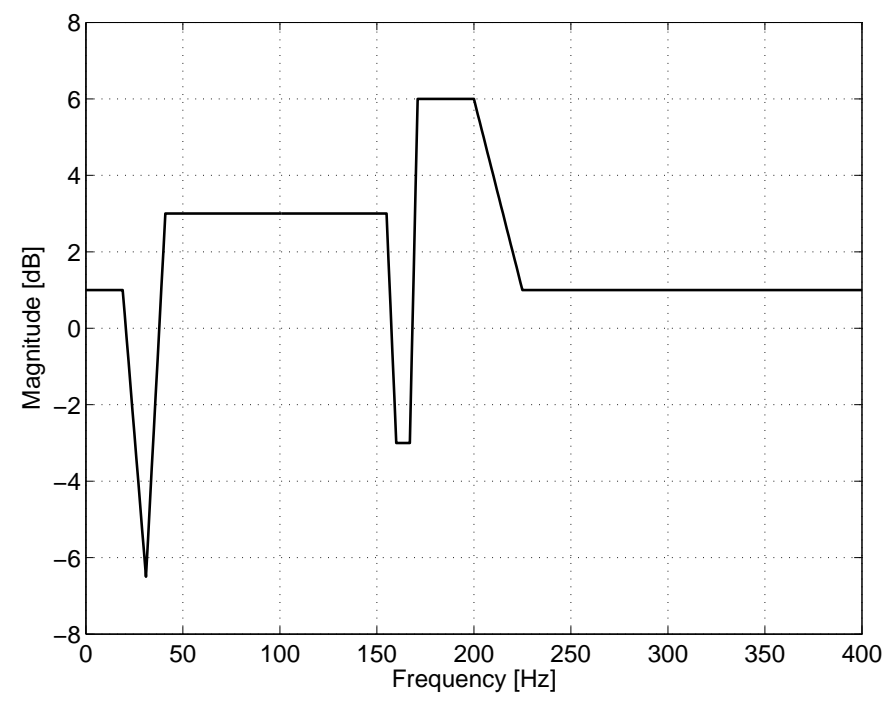

Figure 9: Constraints on the output sensitivity function

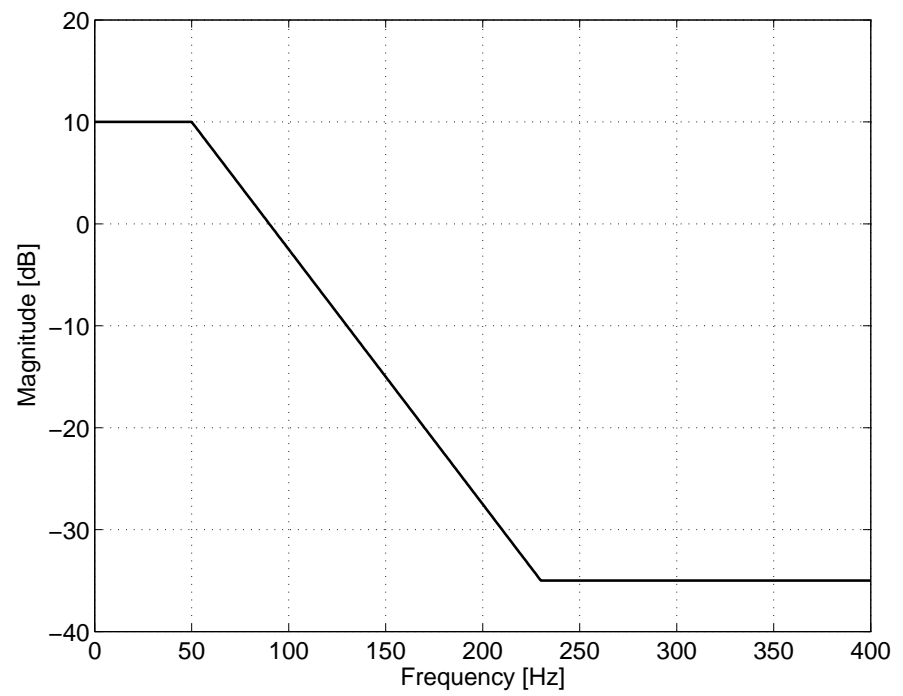

Figure 10: Constraints on the input sensitivity function 


\subsection{Controller Evaluation}

The controllers submitted by the participants have been implemented on the real system. The same PRBS signal have been used as the disturbance for the open-loop and closedloop experiments and the following signals have been measured: the residual forces in open-loop $y_{o l}(t)$ and in closed-loop $y_{c l}(t)$, the input of the primary path $u_{p}(t)$ and the output of the controller $u(t)$.

The magnitude of sensitivity functions are estimated using the spectral analysis method as follows:

$$
\begin{aligned}
\left|S_{y p}\left(e^{j \omega}\right)\right|^{2} & =\frac{\Phi_{y_{c l}}(\omega)}{\Phi_{p}(\omega)} \approx \frac{\Phi_{y_{c l}}(\omega)}{\Phi_{y_{o l}}(\omega)} \\
\left|S_{u p}\left(e^{j \omega}\right)\right|^{2} & =\frac{\Phi_{u}(\omega)}{\Phi_{p}(\omega)} \approx \frac{\Phi_{u}(\omega)}{\Phi_{y_{o l}}(\omega)}
\end{aligned}
$$

where $\Phi_{x}(\omega)$ is the power spectral density of $x$. In practice, since $p(t)$ is not measurable, the output of the system in open-loop operation $y_{o l}(t)$ is used, instead. In order to have a fair comparison a Matlab program is provided in the Benchmark site to compute the estimates of the magnitude of the sensitivity functions.

The controllers are evaluated on the basis of the computation time criterion which is the product of the number of controller parameters and the sampling frequency. However, as all of participants except one, use a sampling frequency of $800 \mathrm{~Hz}$, a normalized criterion is defined :

$$
C_{n}=\left(n_{R}+n_{S}\right) * F_{s} / 800
$$

where $n_{R}$ and $n_{S}$ are the numbers of parameters of the polynomials $R$ and $S$, respectively (note that $n_{R}=n_{r}+1$ and $n_{S}=n_{s}$ ).

\section{Participants}

Several solutions to the benchmark problem have been proposed using different methodologies. In this section the solutions are briefly introduced and are classified from different points of view. A name is given to each design which is related to the affiliation of the corresponding author. Some of the papers are published in this Special Issue and the others can be found in the proceedings of the workshop [15] or in the benchmark site [14]. The solutions are sorted alphabetically as follows:

BORDEAUX [10]: A frequency-domain method based on fractional differentiation is used to compute an initial controller (Crone control). Then the parameters of the controller are tuned to reduce the peak value of power spectral density of the closedloop output by a non-linear optimization algorithm. A discrete-time identified model of the system is used in the control design as well as in the tuning step.

BRESCIA [3]: A non-iterative data-driven controller tuning method is used. In this approach, called Virtual Reference Feedback Tuning (VRFT), the problem of shaping the input and output sensitivity functions is considered as a model reference control problem in two-norm. The controller order is fixed at the beginning and no model of the plant is used in the design. 
CERT [6]: The order of a high-order controller designed based on the $H_{\infty}$ approach is reduced by Robust Modal Control. The dominant poles of the initial controller are chosen as the poles of the reduced-order controller and the parameters of the controller numerator are obtained by minimizing the distance between two controllers, taking into account the closed-loop performance.

CRANFIELD [5]: The minimization of the $l_{1}$ norm of the transfer function between the disturbance and the control input under the sensitivity constrained is considered. A convex optimization approach to this problem leads to a 73th-order controller. Then, this optimal solution is used as a guide-line to design a low-order controller based on a non-convex parameter optimization problem. Finally, it is shown that a second-order controller can achieve almost the same performance.

DELFT [9]: A nonlinear semi-definite programming (SDP) approach to the $H_{\infty}$ fixedorder synthesis problem is proposed. The Newton direction in the optimization problem is replaced by the curved line-search interior point method. The convergence of the proposed approach is also analyzed. The method is compared with two other techniques: a posteriori reduction and the cone complementarity method.

EPFL [12]: A correlation-based data-driven controller tuning method is proposed for the benchmark problem. The idea is to tune the parameters of a fixed-order controller such that the controlled output be uncorrelated with the disturbance signal. The cross-correlation between closed-loop output and the disturbance signal is minimized iteratively using stochastic approximation.

HAMBURG [8]: A reduced-order model of the plant is computed using balanced reduction. Then an $H_{\infty}$ controller with pole region constraint is designed in the continuous-time domain and its order is reduced by zero-pole cancellation. The bilinear transformation is used to obtain a discrete-time controller which violates some constraints. Finally, the controller parameters are fine-tuned manually.

LAG [7]: A high-order controller based on an open-loop identified model is designed using pole placement and sensitivity function shaping by convex optimization. Using this controller, an identification in closed-loop is carried out in order to identify a better design model. Then the controller is re-designed based on the closedloop identified model and implemented on the real system. Finally, the closedloop acquired data are used to estimate a reduced-order controller preserving the desirable properties of the nominal closed-loop system.

MEX [4]: A nonlinear constrained optimization problem is considered for design of a fixed-order controller with a given structure. The authors claim that the parameters of the controller as well as its order are optimized using the genetic algorithm. The performance of the controller is compared with that of an $H_{\infty}$ controller reduced using the balanced truncation method.

SHARIF [2]: A controller order reduction technique is proposed based on the minimization of the nuclear norm (sum of singular values) of a residue matrix related to the controller parameters. In order to ensure the stability of the reduced closed-loop system, some constraints on the difference between the magnitude of the full-order 
and reduced-order open-loop transfer functions are considered. The problem is formulated by Linear Matrix Inequalities and solved using standard tools.

SHEFFIELD [13]: A multi-model approach to reduce controller complexity in model predictive control is used for the benchmark problem. The basic idea is the use of multiple reduced order independent models for one-step ahead up to n-step ahead predictions instead of the recursion on a high-order model to derive the predictions. As MPC is a time-domain approach and is not a natural tool for control problem with frequency-domain specifications, the designed controller for the benchmark problem could not satisfy the specifications.

SUPAERO [1]: An open-loop shaping design on the Nichols plot is performed directly on the non-parametric model of the plant. A simple controller satisfying almost all of the specifications is obtained by trial and errors using specially developed graphical tools.

SUPELEC [11]: The non-convex problem of fixed-order $H_{\infty}$ controller design is considered. An iterative solution based on successive convex optimizations using LMIs and gradient search is proposed to find a local optimum solution. Finally, the parameter of the controller are tuned using the genetic algorithm.

The design methodologies may be classified as follows:

Model-based/Data-Based: The majority of the designs use an identified parametric model of the plant for the controller design. The discrete-time model provided in the benchmark site or a bilinear transformation of this model in continuous-time is employed by CERT, CRANFIELD, DELFT, HAMBURG, SHARIF, SHEFIELD and SUPELEC. Some designers identify their own models from open-loop data (MEX and BORDEAUX) or from closed-loop data (LAG). The controller of BRESCIA is completely model-free and is based only on the plant input-output data. SUPAERO uses a non-parametric model of the plant obtained from spectral analysis. The input-output data are used to tune the controller parameters in the EPFL controller, while a parametric model of the plant is employed for gradient computation. Finally, the closed-loop data are involved in the estimation of the reduced order controller of LAG.

Direct/Indirect approaches: CERT starts with designing a high-order controller based on the high-order model of the plant and then reduces the order of the controller (direct reduction). Some other designs like HAMBURG, LAG and SHARIF use a low-order model of the plant for the high-order controller design and reduce also the order of the controller in the final step (combined direct and indirect reduction). The other approaches compute directly a fixed-order controller from data (BRESCIA, EPFL) or model (BORDEAUX, CRANFIELD, DELFT, MEX, SUPAERO, SUPELEC).

Optimization criterion: CRANFIELD minimizes an $l_{1}$ criterion, BRESCIA, EPFL and LAG which are basically the time-domain approaches try to minimize a twonorm model-following criterion, while DELFT and SUPELEC minimize an $H_{\infty}$ criterion. For high-order controller CERT, SHARIF and HAMBURG use an $H_{\infty}$ 

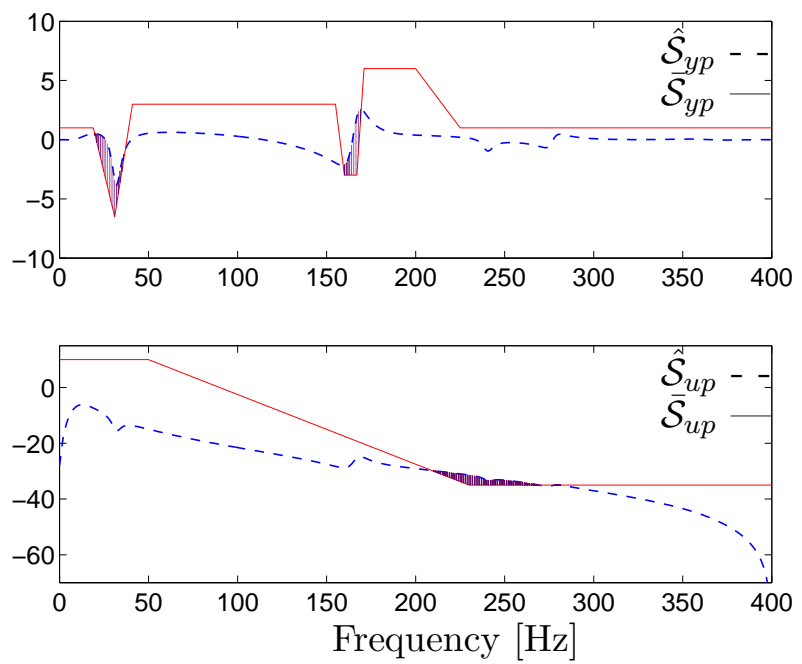

Figure 11: Calculation of the criterion - achieved sensitivity functions $\hat{\mathcal{S}}_{y p}$ and $\hat{\mathcal{S}}_{u p}$ (dashed) vs. constraints $\overline{\mathcal{S}}_{y p}$ and $\overline{\mathcal{S}}_{u p}$ (solid)

criterion, however they use different criteria for controller reduction. CERT minimizes the Frobenius norm and SHARIF the nuclear norm, while HAMBURG uses the balanced truncation technique. A heuristic criterion specially defined for the benchmark problem is minimized by MEX using the genetic algorithm. For the fine-tuning of the final controller BORDEAUX uses the gradient approach and SUPELEC the genetic algorithm. The controllers BORDEAUX and SUPAERO are based on loop shaping in the frequency domain and do not minimize a criterion.

\section{Comparative results}

The comparative results of thirteen solutions to the benchmark problem are presented in this section. The solutions are compared both using the identified model and the real data. Initially, it was expected that the best controller is the lowest-order controller that succeeds in fulfilling the specifications. However, though the specifications are almost met by most of the designs, none has succeeded to fulfill them to a full extent. Therefore, a new comparison criterion has been defined. First, the surface (hatched in Fig. 11) where the estimates of the sensitivity functions $\hat{\mathcal{S}}_{y p}$ (dashed) violate the constraint $\overline{\mathcal{S}}_{y p}$ is calculated:

$$
\Delta \mathcal{S}_{y p}=\sum_{\left|\hat{\mathcal{S}}_{y p}\right|>\left|\overline{\mathcal{S}}_{y p}\right|}\left(\left|\hat{\mathcal{S}}_{y p}\right|_{d B}-\left|\overline{\mathcal{S}}_{y p}\right|_{d B}\right) \Delta f
$$

and similarly

$$
\Delta \mathcal{S}_{u p}=\sum_{\left|\hat{\mathcal{S}}_{u p}\right|>\left|\overline{\mathcal{S}}_{u p}\right|}\left(\left|\hat{\mathcal{S}}_{u p}\right|_{d B}-\left|\overline{\mathcal{S}}_{u p}\right|_{d B}\right) \Delta f
$$

where $\Delta f$ is a fixed integration step. Then, the criterion for comparison is defined as a sum of these two surfaces. Experiments performed on the real active suspension system showed that the presence of the noise is significant on the output of the plant. Thus, to reduce the influence of the noise on the values of the criterion, 5 successive real-time 
Table 1: The normalized controller complexity $C_{n}$ for various designs.

\begin{tabular}{|l|cc|c|}
\hline Participant & $n_{R}$ & $n_{S}$ & $C_{n}$ \\
\hline CRANFIELD & 2 & 2 & 4 \\
EPFL & 3 & 2 & 5 \\
SUPAERO & 4 & 3 & 7 \\
BRESCIA & 7 & 0 & 7 \\
SHEFFIELD & 4 & 4 & 8 \\
SHARIF & 5 & 4 & 9 \\
CERT & 5 & 4 & 9 \\
MEX & 6 & 4 & 10 \\
LAG & 6 & 5 & 11 \\
HAMBURG & 6 & 5 & 11 \\
SUPELEC & 5 & 4 & 11.25 \\
DELFT & 7 & 5 & 12 \\
BORDEAUX & 7 & 6 & 13 \\
\hline
\end{tabular}

experiments have been performed, and the averaged value of the criterion:

$$
J=\frac{1}{5} \sum_{k=1}^{5}\left(\Delta \mathcal{S}_{y p}^{k}+\Delta \mathcal{S}_{u p}^{k}\right)
$$

is considered in the sequel for the comparison. The index $k$ in Eq. 14 denotes the number of the experiment. Whenever the comparison is performed using the identified model the corresponding values have the superscript "m" (for example $J^{m}, \Delta \mathcal{S}_{u p}^{m}, \Delta \mathcal{S}_{u p}^{m}$ ) and when the real data are used the superscript "r" is used.

\subsection{Controller complexity}

The thirteen controllers range from 2 nd order $\left(C_{n}=4\right)$ to 7 th order $\left(C_{n}=13\right)$. Table 1 shows the normalized controller complexity $C_{n}$, as well as the number of parameters of the polynomials $R$ and $S$, respectively $n_{R}$ and $n_{S}$, for various designs. The simplest controller is CRANFIELD, while the most complex one is BORDEAUX. Note that the controller BRESCIA contains only numerator polynomial. All the controllers are designed for the sampling period of $800 \mathrm{~Hz}$ except SUPELEC where the sampling period is $1000 \mathrm{~Hz}$.

\subsection{Criterion (using identified model)}

The identified model used for the comparison in this section is available from the benchmark web site [14]. This high-order model represents approximately the behavior of the plant in open-loop operation and has been used by the majority of the participants. It should be noted that some participants have not used this model in the design, so this comparison may not be completely fair.

Almost all of the designs are close to satisfy the specifications. The minimum of the criterion $J^{m}=0.35$ is provided by CERT. The designs BORDEAUX and SUPELEC provide a small values of $\Delta \mathcal{S}_{y p}^{m}$, however there are a significant violations of the constraint 
Table 2: Criterion calculated using identified model for various designs.

\begin{tabular}{|l|c|cc|c|}
\hline Participant & $C_{n}$ & $\Delta \mathcal{S}_{y p}^{m}$ & $\Delta \mathcal{S}_{u p}^{m}$ & $J^{m}$ \\
\hline CERT & 9 & 0.35 & 0 & 0.35 \\
SUPAERO & 7 & 7.09 & 0 & 7.09 \\
DELFT & 12 & 10.46 & 0 & 10.46 \\
LAG & 11 & 11.46 & 0 & 11.46 \\
HAMBURG & 11 & 17.81 & 0 & 17.81 \\
MEX & 10 & 20.72 & 0 & 20.72 \\
CRANFIELD & 4 & 24.93 & 0 & 24.93 \\
BRESCIA & 7 & 28.29 & 0 & 28.29 \\
EPFL & 5 & 37.54 & 0 & 37.54 \\
SHARIF & 9 & 41.29 & 0 & 41.29 \\
BORDEAUX & 13 & 8.34 & 62.75 & 71.09 \\
SUPELEC & 11.25 & 7.5 & 307.1 & 314.6 \\
SHEFFIELD & 8 & 281 & 2727 & 3008 \\
\hline
\end{tabular}

on $\mathcal{S}_{u p}$ that increase in a large extent the value of aggregate criterion $J^{m}$. Table 2 presents the criterion calculated using identified model for different designs.

\subsection{Criterion (using real data)}

The controller closest to meet the specifications on the real active suspension system is LAG with the aggregate criterion of $J^{r}=9.4$. The designs DELFT, CERT, MEX and HAMBURG provide very close performance. Table 3 gives the criterion calculated using real data for thirteen designs. One can notice that above mentioned controllers which provide a small values of the criterion $J^{r}$ belong to the lower part of Table 1, i.e. they all have a big complexity index $C_{n}$. In the restricted-complexity controller design it is of the interest to make a compromise between these two categories. The value of the criterion $J^{r}$ versus complexity $C_{n}$ for various designs is given in Fig. 12 (note that the values of $J^{r}$ for the designs SHEFFILED, SUPELEC and BORDEAUX exceed the limits shown in the figure).

\subsection{Evaluation}

The different designs are evaluated as follows. In the first phase of the evaluation, among the controllers of the same complexity the one that has the minimal value of the criterion is chosen. The remaining controllers are: CRANFIELD, EPFL, SUPAERO, SHEFFIELD, CERT, MEX, LAG, DELFT and BORDEAUX. Based on the idea that a more complex controller should give better performances, in the second phase we proceed as follows: among the choosen controllers we reject the ones which have greater value of criterion than a lower complexity controller has. For example, controller MEX with the complexity $C_{n}=10$ and $J^{r}=13.92$ is rejected when compared with CERT with $C_{n}=9$ and $J^{r}=13.19$. Therefore, the controllers with acceptable complexity and performance on the real active suspension system are: CRANFIELD, EPFL, SUPAERO, CERT and LAG (see Fig. 13). 


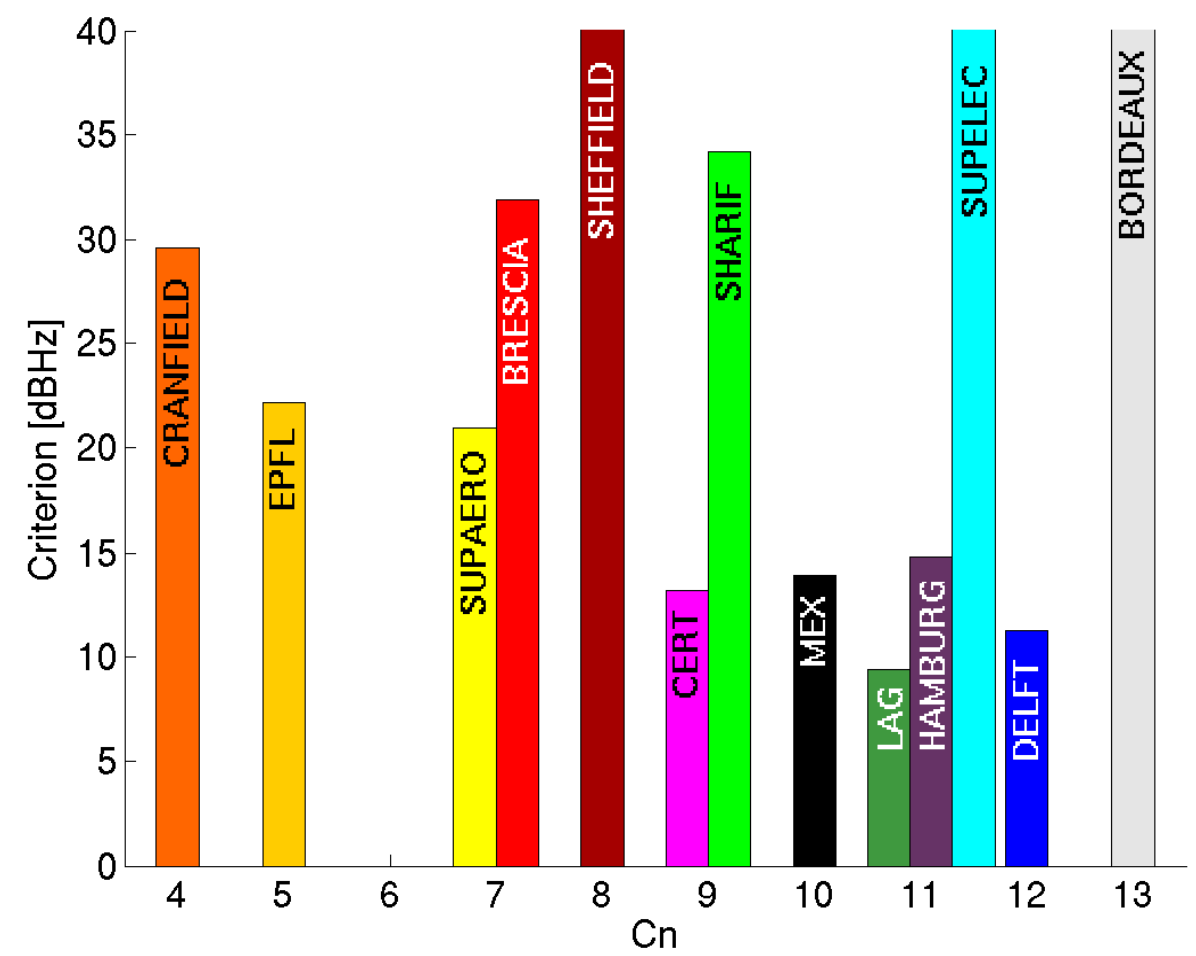

Figure 12: Criterion calculated using real data vs. complexity for various designs

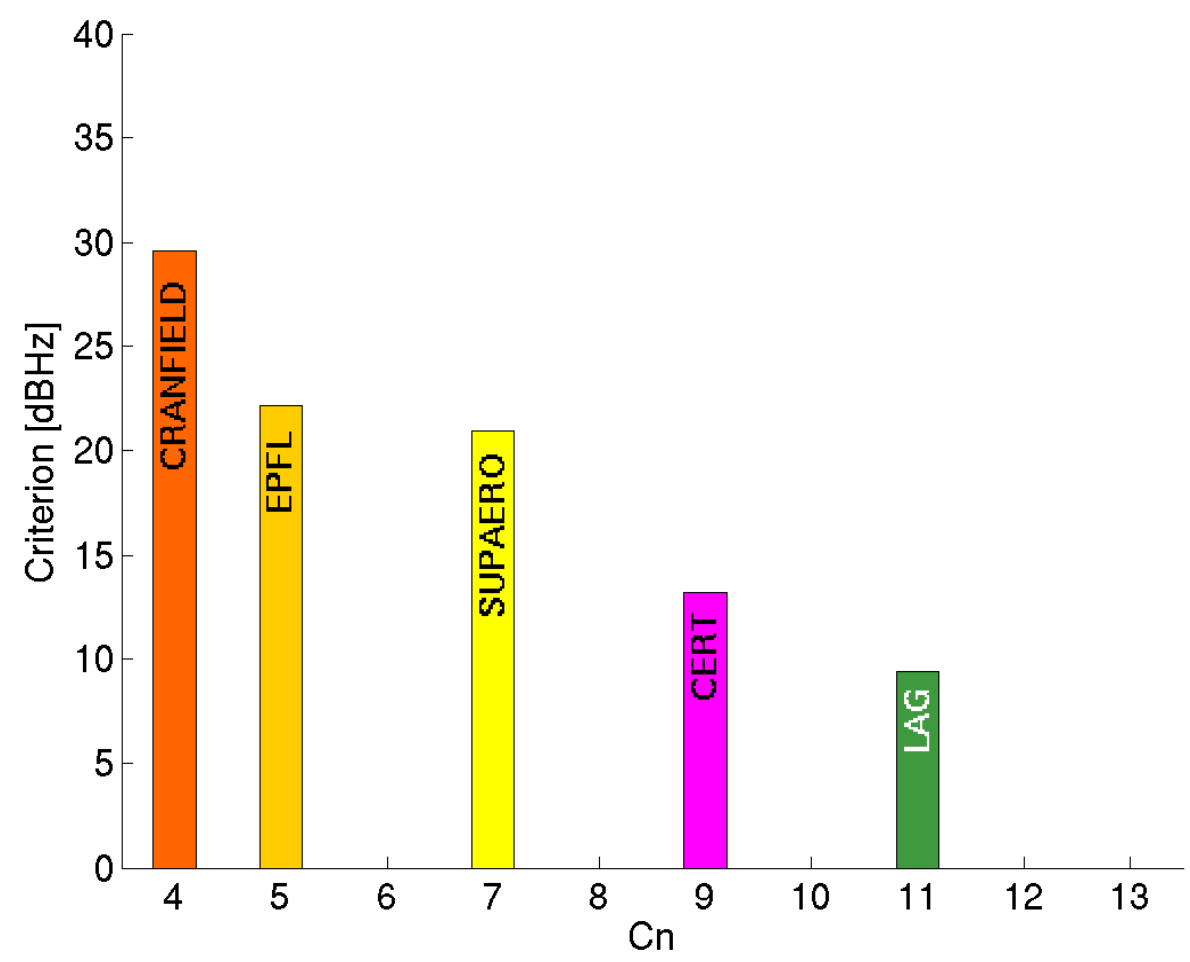

Figure 13: Controllers with acceptable complexity and performance on real system 
Table 3: Criterion calculated using real-data for various designs.

\begin{tabular}{|l|c|cc|c|}
\hline Participant & $C_{n}$ & $\Delta \mathcal{S}_{y p}^{r}$ & $\Delta \mathcal{S}_{u p}^{r}$ & $J^{r}$ \\
\hline LAG & 11 & 9.4 & 0 & 9.4 \\
DELFT & 12 & 11.25 & 0 & 11.25 \\
CERT & 9 & 12.7 & 0.49 & 13.19 \\
MEX & 10 & 13.92 & 0 & 13.92 \\
HAMBURG & 11 & 14.81 & 0 & 14.81 \\
SUPAERO & 7 & 16.82 & 4.09 & 20.91 \\
EPFL & 5 & 22.19 & 0 & 22.19 \\
CRANFIELD & 4 & 25.53 & 4.07 & 29.6 \\
BRESCIA & 7 & 31.92 & 0 & 31.92 \\
SHARIF & 9 & 34.21 & 0 & 34.21 \\
BORDEAUX & 13 & 34.4 & 100.2 & 134.6 \\
SUPELEC & 11.25 & 84.9 & 354.5 & 439.4 \\
SHEFFIELD & 8 & 840.6 & 3260 & 4100.6 \\
\hline
\end{tabular}

\section{Conclusions}

Control of an active suspension system has been considered as a benchmark for restricted complexity controller design. The system has been presented by a set of input-out data and the control evaluation has been performed on the closed-loop data, so the comparison of different solutions was independent of the plant model. Thus, direct and indirect model-based reduction techniques as well as data-driven approaches could be fairly compared. The control specifications have been satisfied more or less by a number of the designs with the controllers range from 2nd-order up to 7 th-order. In general, the methods based on $H_{\infty}$ generated higher order controllers with respect to the methods using direct parameter optimization. The direct use of input-output data in controller design or reduction procedure leads to a good compromise between complexity and performance. Finally one can remark that more than half of the participants did not exploit the data collection possibility offered on the real system and based their design on the provided model . One may ask if the adequate use of the real data would have improved their results.

\section{References}

[1] D. Alazard. Comment on the benchmark for "Design and Optimization of Restricted Complexity Controllers". European Journal of Control, 9(1), January 2003.

[2] R. Amirifar and N. Sadati. A linear matrix inequality approach to $h_{\infty}$ controller order reduction with stability and performance preservation. In International Workshop on "Design and Optimization of Restricted Complexity Controllers", pages 86-91, Grenoble, France, January 2003. 
[3] M. C. Campi, A. Lecchini, and S. M. Savaresi. Tuning of the controller for a benchmark active suspension system through vrft. European Journal of Control, 9(1), January 2003.

[4] D. U. Campos-Delgado, R. Femat, and E. Ruiz-Vélazquez. Design of reduced order controllers via $h_{\infty}$ and parametric optimization: comparison for an active suspension system. European Journal of Control, 9(1), January 2003.

[5] Y. Cao and W. Yan. Low-order controller design using multi-objective optimization. European Journal of Control, 9(1), January 2003.

[6] S. Chable, S. Mahieu, and C. Chiappa. "Design and Optimization of Restricted Complexity Controllers": A modal approach for reduced-order controllers. European Journal of Control, 9(1), January 2003.

[7] A. Constantinescu and I. D. Landau. Direct controller order reducton by identification in closed loop applied to a benchmark problem. European Journal of Control, 9(1), January 2003.

[8] H. Faraz and H. Werner. Reduced complexity control of an active suspension system using $h_{\infty}$ loop shaping and pole region constraints. In International Workshop on "Design and Optimization of Restricted Complexity Controllers", pages 92-97, Grenoble, France, January 2003.

[9] C. W. J. Hol, C. W. Scherer, E. G. Van der Meché, and O. H. Bosgra. A trustregion interior point approach to fixed-order control applied to the benchmark system. European Journal of Control, 9(1), January 2003.

[10] P. Lanusse, T. Poinot, O. Cois, A. Oustaloup, and J. C. Trigeassou. A restrictedcomplexity controller with Crone control-system design and closed-loop tuning. In International Workshop on "Design and Optimization of Restricted Complexity Controllers", pages 116-121, Grenoble, France, January 2003.

[11] F. Le Mauff and G. Duc. Designing a low order robust controller for an acitve suspension system thanks LMI, gradient search and genetic algorithm. European Journal of Control, 9(1), January 2003.

[12] L. Mišković, A. Karimi, and D. Bonvin. Correlation-based tuning of a restrictedcomplexity controller for an active suspension system. European Journal of Control, 9(1), January 2003.

[13] J. A. Rossiter. Using a multi-model to reduce controller complexity in predictive control. In International Workshop on "Design and Optimization of Restricted Complexity Controllers", pages 110-115, Grenoble, France, January 2003.

[14] Benchmark Specifications. Design and Optimization of Restricted Complexity Controllers, Available: http://iawww.epfl.ch/News/EJC_Benchmark/index.html.

[15] International Workshop. Design and Optimization of Restricted Complexity Controllers. Laboratoire d'Automatique de Grenoble, 38402 Saint Martin d'Hères, France, January 15-16, 2003. 